\title{
Fábricas de resiliencia. Una oportunidad para el patrimonio industrial: el caso de La Trinidad
}

Benito Sánchez-Montañés ${ }^{1} \mid$ Manuel V. Castilla ${ }^{2}$

Recibido: 14-01-2020 | en su versión final: 08-04-2020

Resumen

El objetivo de este trabajo es repensar la estrategia para revitalizar los edificios industriales y transformarlos en fuentes de resiliencia, mejorando su vivencia como espacios. Nos centramos en La Fábrica de Vidrio La Trinidad de Sevilla y el estudio de su entorno físico y humano, imprescindible para desarrollar un acercamiento integral. Nos enmarcamos en los campos del patrimonio y del comportamiento ambiental de edificios, en los que se han desarrollado trabajos sobre energía y confort interior. Proponemos un acercamiento a la rehabilitación del patrimonio industrial desde la resiliencia, el diseño ecológico y la interacción social. Como novedad, ampliamos el campo a los requerimientos de una visión necesaria para abordar los retos del futuro inmediato. La importancia de re-habitar el patrimonio industrial se da en una sociedad que necesita mecanismos para enfrentar las mutaciones que pueden aparecer en todos los órdenes. Afortunadamente, estos dos requerimientos se retroalimentan ya que la rehabilitación industrial puede convertirse en una estrategia sostenible clave. La metodología aplica conceptos de diseño ecológico y herramientas para proponer un entorno integrado para el patrimonio. También se plantea como un marco que incluye múltiples herramientas de diseño y datos para analizar. Algunos flujos de trabajo clave se ejemplificarán en el caso elegido. La rehabilitación del patrimonio industrial es una oportunidad para reconstruir el tejido urbano y social, convirtiéndolo en foco de resiliencia para la ciudad. Para ello es necesaria una aproximación con objetivos y herramientas múltiples, como queda ejemplificado para el caso propuesto, puesto que los nuevos retos necesitan un mayor grado de fortaleza, que será fruto de esa red de cualidades en sinergia.

Palabras clave: arquitectura industrial; rehabilitación arquitectónica; construcción ambiental; ecología urbana

Citación

Sánchez-Montañés, B. y Castilla, M. V. (2020). Fábricas de resiliencia. Una oportunidad para el patrimonio industrial: el caso de La Trinidad. ACE: Architecture, City and Environment 15(43), 9192. DOI: http://dx.doi.org/10.5821/ace.15.43.9192

1 Dr. Arquitecto, Profesor Titular de Universidad, Departamento Historia, Teoría y Composición

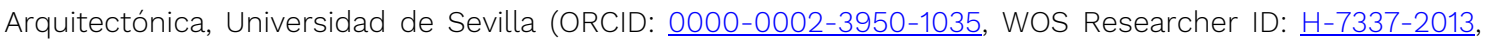
Scopus Author ID: 35253944900); ${ }^{2}$ Dr. Arquitecto, Profesor del Departamento Ingeniería del Diseño, Universidad de Sevilla (ORCID: 0000-0002-4787-5280). Correo de contacto: benitosm@us.es 


\title{
Resilience Factories. An Opportunity for Industrial Heritage: La Trinidad Case Study
}

Abstract

\begin{abstract}
The objective of this work is to rethink the strategy for the revitalization of industrial heritage buildings with the scope of transform them into resilience sources and enhance the architectural experience. We focus in La Trinidad Glass Factory in Seville, to be able to check the social and human environment of a greater interest to develop an integral approach to resilience. Our framework includes the fields of heritage and sustainable behavior of buildings, they had been developed works focused on energy and inner comfort We propose an approach to industrial heritage reuse from resilience, performance of buildings and social interaction. Here, we broaden to the demands of a vision needed for facing the challenges in the foreseeable future. The great importance of re-inhabiting the industrial heritage takes place in a society that needs mechanisms to face mutations that may be looming on all orders. Fortunately, these two statements resonate since industrial retrofitting could become a key strategy for sustainability. This work applies concepts of ecological design and tools to propose a more integrated environment for architectural heritage. The methodology is developed as a framework, which includes multiple design tools and data to be analyzed. Some key workflows will be exemplified by the chosen case. Reliving industrial heritage is an opportunity to rebuild the city fabric, making it a focus of resilience for the city. This requires an approach with multiple objectives and tools as exemplified by the proposed case. Since the new challenges need a greater degree of strength, which will be the result of that network of qualities working in synergy.
\end{abstract}

Keywords: industrial architecture; architectural reuse; environmental construction; urban ecology

\section{Introducción y objetivos de la investigación}

Esta investigación se ha concretado tras los debates mantenidos durante una reciente oportunidad en Sevilla para reflexionar sobre la reutilización de la arquitectura del patrimonio industrial, bajo la forma de una reunión académica.

La ciudad que hemos heredado es un palimpsesto en el que los estratos del pasado constituyen una parte fundamental de la trama física y prácticamente toda su memoria e identidad (Campos-Sánchez et al., 2018). Esto es así en aquellas partes del mundo donde los humanos han perseverado en sus asentamientos, como es habitual en el caso de Europa. Así, una parte de la ciudad construye la dimensión material que llamamos "patrimonio", cuya definición resulta de la construcción de sucesivas Cartas que han sido producidas durante el Siglo XX, (Atenas, Venecia, Nara, Cracovia...) y que han generado la idea de "valor cultural" con el que ahora reconocemos el extenso hecho patrimonial.

Una parte no pequeña del trabajo de edificación que hoy se realiza, se desenvuelve, de una u otra forma, relacionado con ese patrimonio, en un campo abierto que damos en llamar "arquitectura y memoria”. Este término es más amplio que el de rehabilitación, o el de restauración, o varios otros que se han forjado, y nos interesa esa capacidad de apertura. Dentro de los márgenes de la memoria

ACE, 15 (43) CC BY-ND 3.0 ES | UPC Barcelona, España | Fábricas de resiliencia. Una oportunidad para el patrimonio industrial: el caso de La Trinidad. DOI: http://dx.doi.org/10.5821/ace.15.43.9192 
construida encajan un creciente número de objetos y realidades. Estos márgenes pueden definirse desde distintas disciplinas, como hemos apuntado. Las culturas que miran a su pasado van asumiendo paulatinamente la riqueza y complejidad del mismo, reflejada en innumerables manifestaciones que se entretejen para formar la matriz que constituye la existencia humana y su decurso en el tiempo. Como consecuencia, el valor patrimonial impregna cada vez a más actividades, procesos, herramientas, fenómenos, objetos muebles e inmuebles. En esta realidad, la idea de "patrimonio industrial" se ha consolidado, y son numerosos los autores que han tomado parte en el desarrollo de esta definición, entre los cuales mencionaremos a Sobrino (2010), del que hemos obtenido la definición operativa de "paisaje de producción”. Esta definición recoge y explica la realidad de muchas ciudades, cuyo tejido está construido, soportado y modificado por este componente productivo, patente en estructuras industriales, de transporte, abastecimiento, comercialización, que constituyen la nómina del mencionado patrimonio industrial... Es este marco conceptual el que hemos elegido para la presente reflexión. Actualmente, con la desindustrialización, muchos han derivado en ruinas industriales. Su valor va mucho más allá de meros contenedores, puesto que conforman la expresión del trabajo y del lugar. Entidades como TICCIH, ICOMOS y UNESCO promueven acciones para reconocer, mejorar y divulgar este material como una parte de la memoria urbana.

Es bien sabido que las llamadas ciudades desarrolladas acumulan gran cantidad de estos contenedores industriales que han quedado obsoletos, lo que es un hecho con trascendencia desde la escala del edificio hasta la metropolitana. Por lo tanto, la gestión/intervención de estos edificios es de enorme importancia para la evolución de su entorno inmediato y de la ciudad en su conjunto (Sobrino, 2015 y Oglethorpe, 2013). La industrial ha sido, en muchas ocasiones, un serio problema para el medio ambiente, que ha afectado al ecosistema inmediato y remoto. Por ello, la sostenibilidad y la resiliencia del paisaje de producción pasa por considerar la intervención en estos edificios con los criterios adecuados, de forma que transforme su carácter de problema en el de centro de sostenibilidad de su entorno, como ejemplifica el profesor Kevseroğlu (2014) en su trabajo dedicado que explora la integración urbana del patrimonio industrial. Este objetivo implica el conocimiento de estrategias que produzcan el correcto comportamiento ambiental de un edificio de estas características. Por ello, es necesario añadir el concepto de "inteligencia ecológica” de la arquitectura al estudio detallado de las peculiaridades de los contenedores productivos, para identificar la oportunidad que encierran al servicio de las comunidades que los han levantado. Se puede afirmar que este enfoque se alinea con el hecho de que la rehabilitación es la actuación más sostenible posible en la construcción, como ha sido sobradamente demostrado, por ejemplo, por la profesora De Luxan en su estudio sobre buenas prácticas en rehabilitación (Ayuntamiento de Madrid, 2010), por ejemplo, afirma que el ahorro de energía y emisiones es aproximadamente del 60\% si comparamos la rehabilitación integral de un edificio con la construcción de otro nuevo equivalente. No se está afirmando que cualquier tipo de reestructuración de un edificio existente es necesariamente una intervención realmente sostenible. Deben integrarse una serie de aspectos tales como materiales adecuados, consideraciones energéticas durante la obra y su uso posterior y una estrategia cuyo objetivo principal sea el impacto ambiental positivo, donde toda intervención se convierta en un activador ecológico. Esto aseguraría que el patrimonio industrial se convirtiese en una oportunidad para mejorar nuestro medio habitado y enfrentar el futuro con posibilidades de éxito.

Tal y como hemos desarrollado en investigaciones anteriores (Sánchez-Montañés, 2011 y Castilla, 2016), una aproximación al comportamiento ambiental de la arquitectura heredada nos permite poner en valor nuestro patrimonio desde un campo muy poco explorado, lo que constituye una herramienta eficaz para re-habitarlo. El análisis bioclimático de la arquitectura heredada del pasado implica comprender la interacción del edificio con el clima, como sabemos desde que Givoni (1969) sentó las bases del diseño bioclimático. Es necesario diseñar técnicas y formas de acondicionamiento natural que no precisen de un sistema artificial como soporte (Iwaro \& Mwasha 2014). Esta investigación

ACE, 15 (43) CC BY-ND 3.0 ES | UPC Barcelona, España | Fábricas de resiliencia. Una oportunidad para el patrimonio 
pretende ayudar a demostrar que el patrimonio arquitectónico tiene un valor intemporal que debe ser respetado y mantenido. Este valor es el de interactuar de manera positiva con el entorno.

No obstante, la arquitectura industrial se encuentra, en muchos casos, en una situación distinta. La que se ha construido desde mediados del Siglo XIX y principalmente en el Siglo XX, ha sido frecuentemente realizada con absoluta indiferencia hacia el clima y sus propias necesidades de confort, como ha sido cuantificado por Watson en The TICCIH Guide to Industrial Heritage Conservation (2012). También podemos argumentar que tienen que ver con la esencia del impulso "maquinista" de la idea misma de la modernidad que impregnaba esos tiempos y, por supuesto, con su vocación internacionalista (Trachana. 2008). En estos casos, no es necesario hablar sobre la recuperación de los valores ambientales de estos edificios. Aplicando inteligencia ambiental, encontraremos procesos que reviertan el comportamiento del contenedor construido, facilitando una relación más armoniosa con su entorno. Como Landorf (2009) ejemplifica con detalle, es necesario establecer criterios bioclimáticos para actuar sobre el patrimonio de forma que la intervención sea realmente sostenible, buscando la resiliencia como objetivo. Quizás todavía sea necesario recordar que, en la situación actual, la sostenibilidad no es un objetivo suficiente. Podemos afirmar con Hopkins (2008) en el texto fundacional de la Red de Transición y de manera más sistemática pero menos explícita con Fiksel (2006) que la sostenibilidad es en este momento un objetivo inalcanzable, ya que nuestra especie ha superado el punto de la curva de impacto global donde la insostenibilidad aún era reversible. Sin embargo, nos desplazamos hacia un escenario en el que el objetivo es construir un hábitat resiliente, capaz de soportar las modificaciones de los parámetros del entorno que permita mantener la vida humana en la Tierra con niveles razonables de viabilidad y bienestar. Este objetivo trata de hacer frente a las nuevas condiciones físicas y la realidad de los recursos. Hay que señalar que esto implica condiciones objetivas, medibles. Tal marco conduce a un enfoque que debe incluir un método integral donde el conocimiento y los datos científicos encuentren su lugar y den indicaciones precisas sobre la toma de decisiones.

\section{Método}

Los antecedentes y el desarrollo de un método aplicable al proceso de diseño de intervención patrimonial se publicaron en (Castilla, 2018). En él se presenta un conjunto detallado de herramientas utilizables en el proceso de diseño de una intervención en el patrimonio, que actúan en un entorno modular y eficiente como parte integral de un ambiente de trabajo colaborativo. Así, esa metodología ha de considerarse como una base para el trabajo presentado aquí. En el contexto descrito, arquitectos e ingenieros, se encuentran frecuentemente en el papel de mediadores entre los inversores, la comunidad científica, la administración patrimonial y el público en general. Falta una interacción entre ellos. El resultado de la actividad arquitectónica es crucial, no sólo en términos de diseño, sino también de un impacto significativo en las oportunidades para aumentar la conciencia sobre el objeto. En estas circunstancias, la transferencia de resultados de la investigación y el resultado de disciplinas orientadas hacia el futuro requiere nuestra atención.

De forma general, el establecimiento de protocolos adecuados a cada situación y clima específicos genera resultados inteligibles sobre el comportamiento ambiental de la arquitectura (Attia et al., 2012). En consecuencia, es necesario determinar objetivamente los valores ambientales de esta arquitectura histórica para su preservación. Necesitamos proporcionar un conocimiento científico objetivo aplicado a cada caso de estudio, que nos permita mostrar el funcionamiento ambiental de estos elementos de la historia industrial, superando las habituales declaraciones confusas, carentes de datos contrastados. Por lo tanto, la interacción entre un equipo colaborativo a través de la metodología sugerida se considera fundamental en este nuevo escenario. En nuestro caso, partiendo de lo anteriormente expuesto, el método utilizado se basa en tres fases principales: una fase de

ACE, 15 (43) CC BY-ND 3.0 ES | UPC Barcelona, España | Fábricas de resiliencia. Una oportunidad para el patrimonio industrial: el caso de La Trinidad. DOI: http://dx.doi.org/10.5821/ace.15.43.9192 
conceptualización de la identidad patrimonial, un análisis simulado multicapa y una fase de materialización (propuesta). De esta forma, cualquier entorno integrado puede ser visto como una interacción de diferentes multiherramientas básicas y datos formando parte de un DSS, Decision Support System - Sistema de Soporte a Decisiones (Figura 1).

Figura 1. Esquema general del método

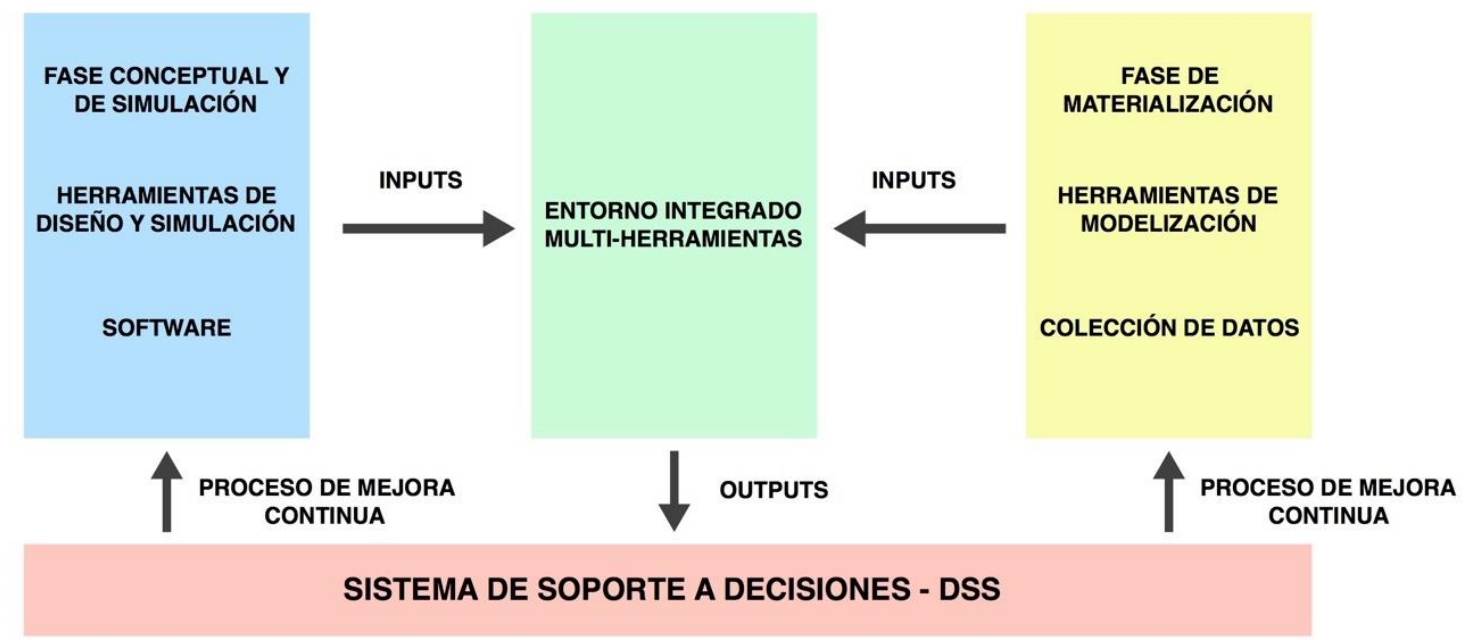

Fuente: elaboración de los autores.

El análisis a través de la simulación generará datos para tomar decisiones relacionadas principalmente con la forma del edificio, su materialidad y distribución funcional. Las fases de conceptualización y materialización (proyecto e intervención) pueden incluir la familia de decisiones sobre una concepción más amplia acerca de la futura vida del edificio, qué significa para sus usuarios y en muchos casos, para el entorno (barrio) circundante. Todo el conjunto de decisiones determinará la resiliencia de la operación resultante.

Las ventajas de este modelo propuesto incluyen:

- Un enfoque multiherramienta que puede proporcionar modularidad, flexibilidad y extensibilidad en términos de control de funcionamiento y herramientas de comportamiento del sistema.

- Un soporte de varios usuarios que ejecutan simultáneamente múltiples tareas de diseño en diferentes proyectos.

- Dividir la funcionalidad entre múltiples herramientas específicas aumentará la eficiencia del sistema.

- Diversas herramientas pueden mejorar la dinámica del entorno de software integrado.

Considerando que en la investigación anterior era factible la metodología relacionada con los criterios bioclimáticos y la simulación, la realidad extremadamente diversa del patrimonio industrial, en términos de la naturaleza de la edificación, situación urbana (o no) y relación con el ámbito social, hace casi imposible establecer un método preciso en relación con todas las consideraciones posibles sobre la resiliencia social y ambiental. Debe desarrollarse una aproximación específica para cada caso, teniendo en cuenta al menos los siguientes criterios principales:

- Autosuficiencia para el propio edificio y la red social relacionada con él. Esta autosuficiencia puede incluir términos como energía, agua, mercancías, alimentos y otros dependiendo del caso.

- Reducción del impacto ambiental de la operación a corto y largo plazo. Incluyendo herramientas de control como LCA (Life Cycle Assessment - Análisis del Ciclo de Vida), huella ambiental o la más adecuada para el caso.

- Contribución a la gestión de residuos, permitiendo un ciclo lo más cercano posible para cualquier tipo de residuo. 
- Acciones para mitigar las consecuencias del cambio climático. Esto dependerá de muchos factores locales. Puede ser la lucha contra el efecto isla de calor urbana (ICU, como se ejemplifica en este caso), planeamiento para situaciones de inundación, sequía severa u otras muchas situaciones posibles.

- Movilidad sostenible. Integración del proyecto en un concepto territorial en el que se reducen las necesidades de desplazamientos diarios y se promueven medios de transporte colectivos y/o sin energía.

- Reforzamiento de la red social de proximidad. Mejorar los medios para reunirse, intercambiar, tejer la red humana en la proximidad. Con el fin de construir una dimensión social del barrio más resiliente.

Esta lista de objetivos debe ser un mínimo exigible. Aunque habrá casos en los que surgirán otros criterios como objetivos básicos para la resiliencia local. Como ejemplo de aplicación del flujo de trabajo con la metodología propuesta (Figura 1) analizaremos nuestro caso de estudio en la sección siguiente.

\section{La fábrica de vidrio de La Trinidad. Sevilla}

La Fábrica de Vidrio de La Trinidad es un complejo industrial de principios del siglo XX que, debido al crecimiento de la ciudad, se ha insertado dentro de la ciudad histórica consolidada, siendo un ejemplo de relación indisoluble entre ciudad e industria. No es objeto del presente trabajo justificar el valor patrimonial del propio edificio y de su estructura productiva y social relacionada, puesto que es algo que ya ha sido suficientemente hecho. Prácticamente desde su cierre y más en concreto desde los últimos años del siglo pasado, vienen desarrollándose acciones sociales e investigaciones académicas encaminadas a poner en valor los significados arquitectónico y etnológico del conjunto. Como resultado de ello, cuenta con la declaración de BIC (Bien de Interés Cultural) como lugar de interés etnológico, según ORDEN de 4 de septiembre de 2001, de la Consejería de Cultura de la Junta de Andalucía', en la que se inscribe el bien en el Catálogo General del Patrimonio Histórico Andaluz y en cuyo anexo quedan descritos profusamente los elementos y valores de la fábrica. Se ha señalado repetidamente que, a pesar de tratarse de una declaración de bien etnológico, el expediente incoado hace hincapié fundamentalmente en el elemento arquitectónico (Sobrino, 2018). Por otro lado, en esta misma obra citada, se hace el más extenso análisis de los valores patrimoniales de la fábrica, además de recoger en su bibliografía una prolija recopilación de los estudios y análisis patrimoniales dedicados a esta pieza y a su entorno material e inmaterial (etnológico). A modo de resumen de los valores puramente arquitectónicos, podemos reseñar que la fábrica se constituye en una serie de naves diáfanas productivas, entre las que destaca una principal, sobre un basamento de espacios técnicos y auxiliares. Es importante su chimenea con depósito de presión, como hito en el entorno urbano, así como el recinto de la fábrica y los edificios auxiliares de acceso, que caracterizan un conjunto cerrado con autonomía respecto al entorno. De hecho, el mantenimiento de esta separación y del aislamiento del conjunto ha sido uno de los debates patrimoniales que se reflejan en los documentos citados y que se considerarán en los ejemplos de nuestro trabajo. Como lenguaje arquitectónico, las piezas principales de la fábrica se definieron en estilo regionalista poco marcado, propio de la arquitectura fabril de la época en Sevilla, en el que se desarrolló un estilo con referencias a la arquitectura local imperante para cualquier construcción de prestigio. Si bien en estos casos se aplicaba una sintaxis simplificada y económica, sólo en los elementos más significativos (básicamente en la fachada principal) como en el caso que nos ocupa.

Hay que resaltar también que el mantenimiento y puesta en carga de La Trinidad tiene el apoyo de una plataforma social que promueve la reactivación del barrio y su reconversión como museo del

${ }^{1}$ Disponible en https://www.juntadeandalucia.es/boja/2001/110/50 
vidrio y centro cívico. Más adelante se debatirá sobre aspectos inmateriales del valor patrimonial del conjunto, su imbricación con el tejido y devenir social del barrio, que son fundamentales, ya que su declaración como BIC tiene carácter etnológico y, por tanto, es imposible valorar la fábrica sin considerar esta dimensión. Este marco lo convierte en un ejemplo ideal para estudiar la fase urbana/social del enfoque propuesto.

Para esta intervención y reutilización del patrimonio industrial, hemos utilizado los siguientes grupos de datos y herramientas como estrategia resiliente:

- Herramientas relacionadas con el contexto urbano/social en la fase de conceptualización: Integración conceptual en el espacio urbano. Antecedentes históricos y contexto actual dentro de la ciudad; integración local y global; plan de ejes directores; estructuras sociales y económicas; evolución del barrio.

- Herramientas relacionadas con la simulación: Software específico; criterios bioclimáticos; resiliencia óptima.

- Herramientas relacionadas con el proceso de materialización: Materiales, soluciones, claves de intervención.

De acuerdo con estos tres grupos de herramientas, proponemos los siguientes pasos de evaluación.

\subsection{Integración conceptual en el espacio urbano y la red social}

Las propuestas de reutilización y protección patrimonial del complejo fueron algunos de los puntos de partida de los diferentes trabajos llevados a cabo por el grupo de investigación. Los trabajos se complementaron con visitas y trabajos de campo que permitieron la comprensión de la ciudad, así como la metodología de trabajo.

Los contextos históricos y urbanos son esenciales para la intervención sobre cualquier elemento arquitectónico. Como resultado de esta fase del estudio, los barrios circundantes se definieron en su forma, densidad y posición, estrechamente vinculados a la existencia y desarrollo de la Fábrica, como podemos ver en la Figura 2. El declive y el cierre final de la misma fueron razones clave para la decadencia social y los problemas de dichos barrios, como ha desarrollado de forma genérica Bender (2001). El componente etnológico, productivo y social de una investigación de estas características debe tener en cuenta la importancia de tales edificios industriales para configurar la identidad de un territorio, sea urbano o no. Un tratamiento consciente asegurará que después de la rehabilitación, sus usuarios sigan percibiendo estas arquitecturas como propias (Andrade y Bragança, 2011).

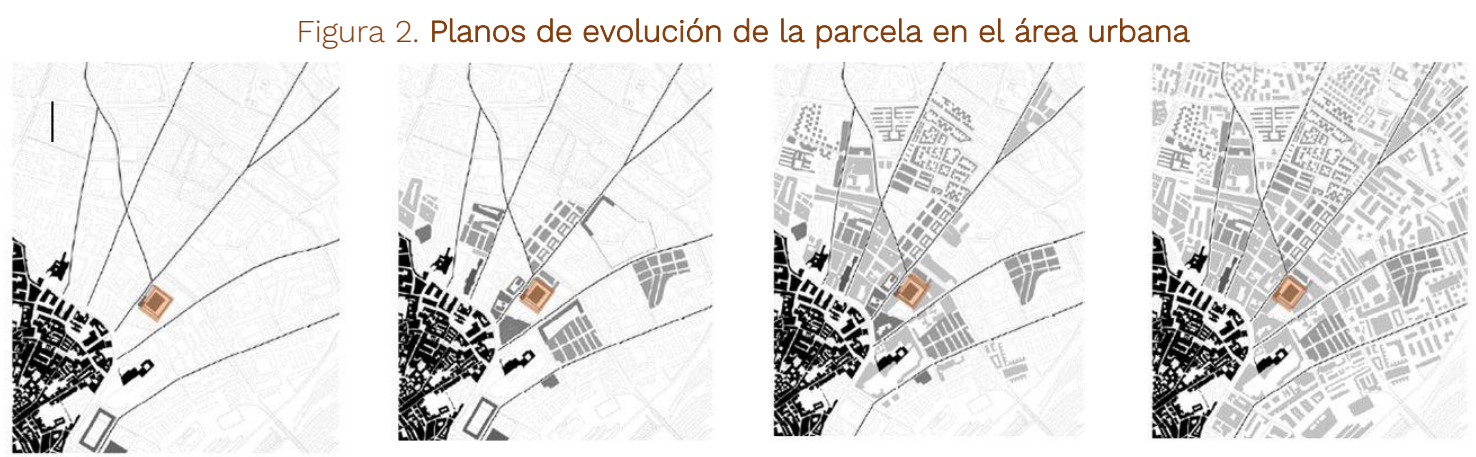

En naranja, la fábrica y cómo los nuevos crecimientos, en gris, fuera de la ciudad amurallada, en negro, la están absorbiendo. Fuente: Sobrino (2018).

El proyecto en sí es una herramienta de simulación marco, donde integramos el conocimiento de la realidad urbana que se ha generado en torno a la fábrica y cómo modifica y condiciona las relaciones

ACE, 15 (43) CC BY-ND 3.0 ES | UPC Barcelona, España | Fábricas de resiliencia. Una oportunidad para el patrimonio industrial: el caso de La Trinidad. DOI: http://dx.doi.org/10.5821/ace.15.43.9192 
que tienen lugar a su alrededor. En la Figura 3 podemos ver cómo una de las principales claves para la intervención es su reintegración en la red urbana, fomentando las conexiones y creando dentro un núcleo de vecindad de interacción social. Esta interacción social debe ser promovida por los diferentes programas que se pretende que sean alojados en la fábrica.

Figura 3. Diagrama de flujo de conexiones de barrio en el área de estudio
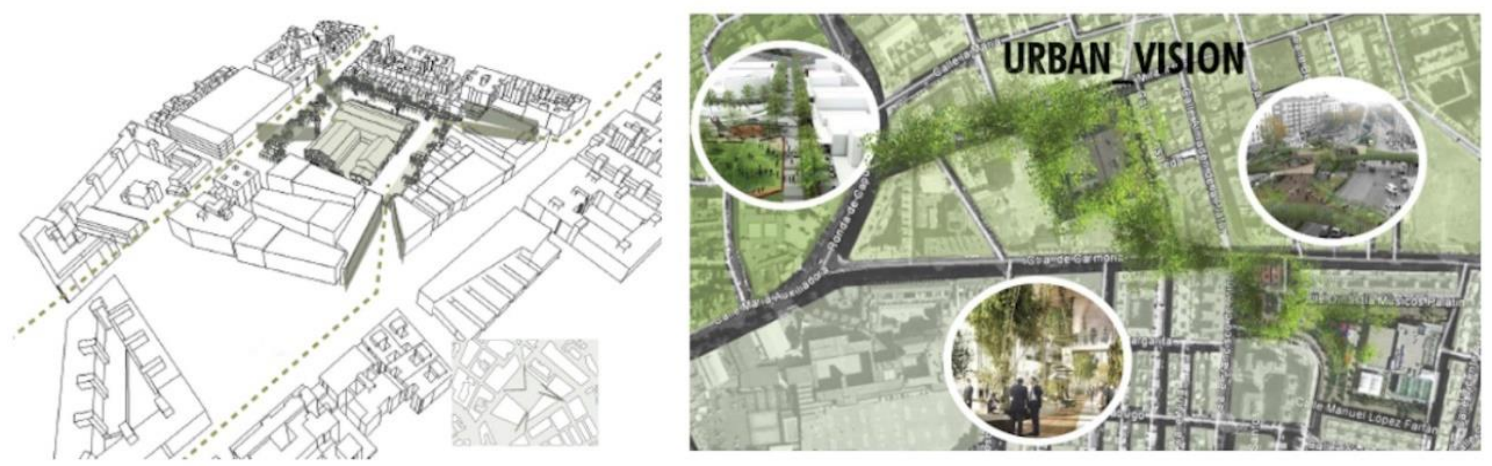

Fuente: Elaboración propia de los autores y del equipo de investigación participante.

La revalorización del elemento arquitectónico representa una parte importante del trabajo desarrollado. A pesar de la dificultad que presentan los edificios industriales debido a su morfología, asociada a funciones muy específicas, es necesario recuperar esta realidad más allá de su uso como contenedores gigantes, no cayendo en la idea de Caja Blanca, metáfora acuñada por primera vez por Hernández (2007).

Un pilar esencial es la conversión de la fábrica en un punto de generación de resiliencia ambiental, aportando así valores que podrían servir de ejemplo para la estructura de transición ecológica del barrio y la ciudad (Lombera y Rojo, 2010). Una de las herramientas adecuadas a este objetivo es la evaluación del Ciclo de Vida mediante el mencionado LCA, ya que es la principal herramienta disponible para cuantificar objetivamente el coste ambiental de cualquier operación arquitectónica. Podemos asumir que, en la situación actual, el LCA demuestra cómo la rehabilitación es una acción mucho respetuosa con el medio ambiente que el levantamiento de un nuevo edificio equivalente (Vilches et al., 2016). Nuestro enfoque es considerar que la fábrica tiene que comportarse de nuevo como un centro de producción, pero esta vez centrada en la energía limpia y la absorción de CO2, contribuyendo a la reducción del efecto Isla de Calor Urbana, según definición de Manley (1958).

\subsection{Fase de simulación de la Fábrica de Vidrio de La Trinidad}

Esta fase se apoya en herramientas relacionadas con la simulación desde dos enfoques con objetivo ambiental: el software bioclimático y la integración de proyectos.

\subsubsection{Simulación bioclimática}

Es ampliamente aceptado que la gestión de la radiación solar es una herramienta fundamental para el rendimiento bioclimático en latitudes y climas como el de Sevilla (Rubio et al., 2015). El objetivo es evaluar el diseño de la Fábrica de Vidrio de la Trinidad para cuantificar cómo se ve afectada por la insolación y proporcionar información sobre las características del diseño que afecta la eficiencia, el confort para posibles usos y la conservación. Especialmente, se examinarán las estrategias de diseño, como la orientación y la protección solar, además del efecto sobre la iluminación natural, sombreamiento y ganancia térmica. Aunque se ha investigado mucho sobre estos aspectos, nos referiremos aquí a tres textos clave, específicamente utilizados en nuestro trabajo, los de Minslow et

ACE, 15 (43) CC BY-ND 3.0 ES | UPC Barcelona, España | Fábricas de resiliencia. Una oportunidad para el patrimonio industrial: el caso de La Trinidad. DOI: http://dx.doi.org/10.5821/ace.15.43.9192 
al. (2001), Higón (2003) y Fiocchi et al. (2011). Este análisis se basa en la metodología desarrollada para aumentar herramientas y datos en un proceso colaborativo para el patrimonio industrial.

La edificación en nave única de grandes dimensiones es característica de las fábricas de usos múltiples en ese momento (principios del s. XX). Este edificio presenta una cubierta a dos aguas, discontinuas ambas a efectos de iluminación y ventilación, sobre una estructura ligera de hierro que se apoya sobre pilares embebidos en los muros de fábrica con grandes ventanales en ambos hastiales. Figura 4. Sobre los aspectos patrimoniales y estilísticos, nos remitimos a lo ya dicho en la introducción de este apartado. Cabe añadir que la construcción es básicamente de paramentos ciegos, con huecos que se recortan en los mismos, heredera de una arquitectura doméstica de prestigio. Esta concepción incide en los niveles de iluminación natural de la fábrica, cuestión que se analizará a continuación como parte fundamental de la simulación bioclimática. Frente a este esquema, otros modelos mucho más acristalados de arquitectura (bien por fachada, bien por la cubierta) se comenzaban a ensayar en otras partes de España (especialmente en Cataluña) y sobre todo en Centroeuropa. Estos modelos acabarían constituyendo el sistema compositivo de referencia en la arquitectura fabril, debido a razones funcionales, vinculadas a las necesidades de iluminación, aunque también respondiendo al deseo de elaborar un lenguaje expresivo propio que reivindicase la presencia de la industria a través de sus contenedores.

Figura 4. Fábrica de Vidrio de la Trinidad
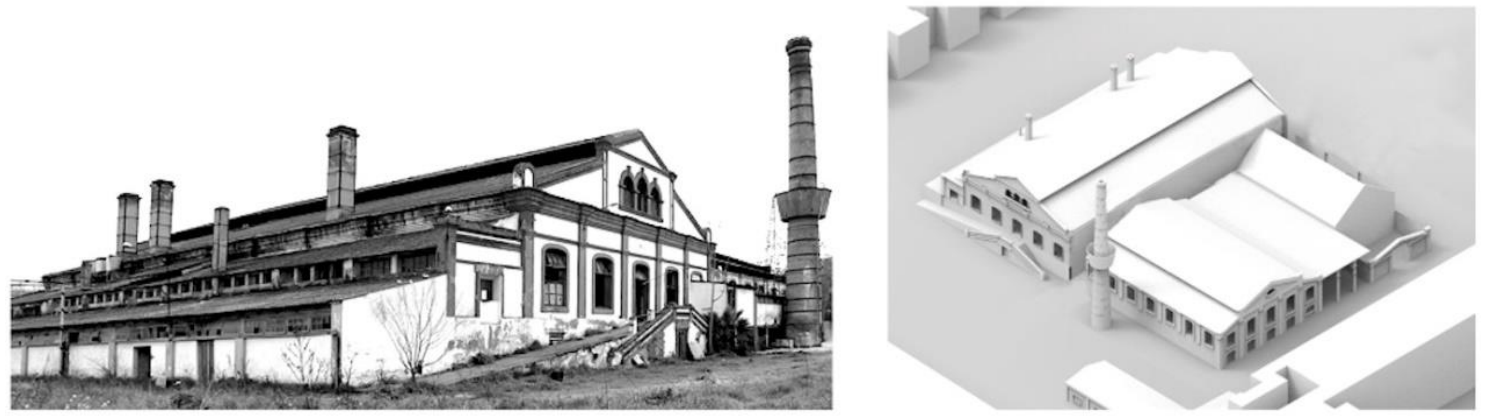

Fuente: Autores

\subsubsection{Clima}

Comprender las condiciones climáticas contextualiza el análisis de esta investigación. Sevilla es considerada una ciudad con clima templado-cálido debido a su latitud y entorno. La temperatura media anual es de 19,2ํ y tiene oscilaciones de temperatura moderadas en períodos fríos y cálidos. Los inviernos son suaves, con una temperatura mínima media no inferior a 5드, y veranos muy tórridos más largos y calurosos cada año (las temperaturas máximas extremas se pueden dar desde el mes de mayo hasta el mes de septiembre). Según la AEMET (Agencia Estatal de Meteorología), rara vez se han observado en la zona temperaturas por encima de los 46ㅇ․ No obstante, desde 2015 se han registrado en cinco ocasiones temperaturas superiores a los 45으, siendo cada vez más frecuente el registro de muy altas temperaturas.

\subsubsection{Datos solares y simulación}

La luz natural es un factor clave que afecta directamente a la componente térmica y al confort; por lo tanto, su consideración es un paso inicial básico en la arquitectura, usando la metodología apropiada de simulación y no sólo datos genéricos. La Trinidad tiene orientación Noroeste en su fachada principal y Sureste para la trasera, lo que deja un lado de la cubierta con una alta exposición al Sur. Las coordenadas son 37,4N / 5,9ㅇ․

ACE, 15 (43) CC BY-ND 3.0 ES | UPC Barcelona, España | Fábricas de resiliencia. Una oportunidad para el patrimonio industrial: el caso de La Trinidad. DOI: http://dx.doi.org/10.5821/ace.15.43.9192 
Para la aplicación del método sugerido, la Figura 5 resume los parámetros más relevantes (herramientas y datos) implicados en el proceso de multiherramientas mencionadas anteriormente (Olmos, 2012 y Rosen, 2012). Herramientas seleccionadas de la Figura 5 nos permiten estimar los datos de energía solar, la insolación y la iluminación durante un período de tiempo específico con precisión, proporcionando información sobre características del diseño que influirán de manera eficiente y pueden ayudar a su correcto funcionamiento y, con él, a su preservación en su nueva etapa. Concretamente para el presente estudio se utiliza la herramienta Radiance incorporada en ECOTEC de AutoDesk, según los estándares y los archivos climáticos aceptados por defecto para los parámetros analizados (Wood, 2010).

Figura 5. Herramientas de diseño de las fases conceptual y de simulación
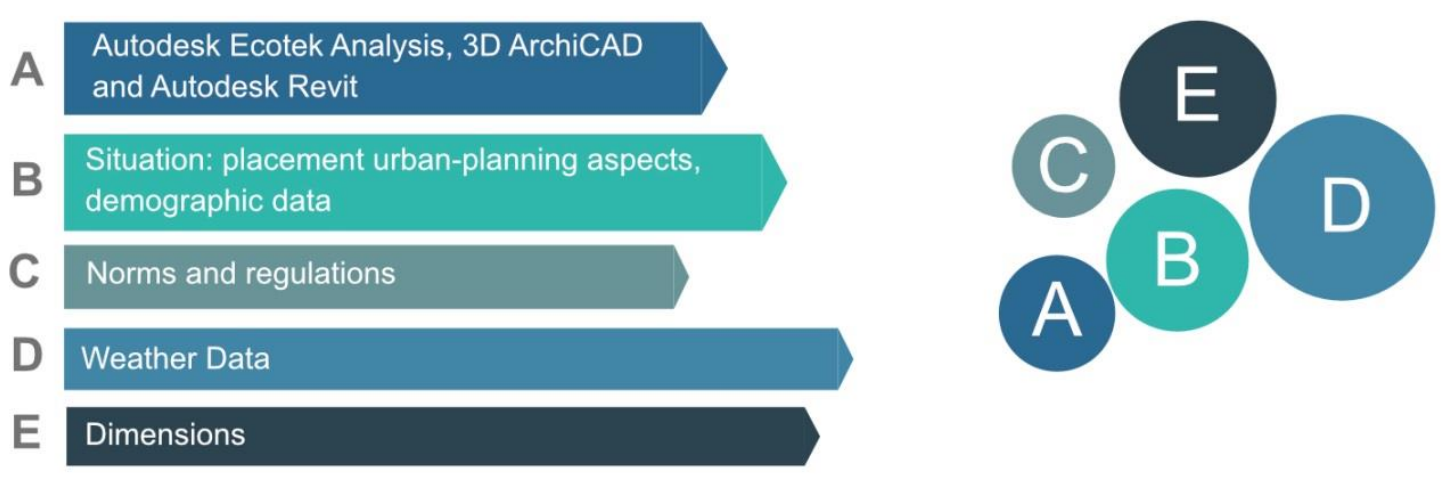

Fuente: Autores.

\subsubsection{Resultados de la simulación en la Fábrica de Vidrio de La Trinidad}

Hay que destacar la importancia de la interacción de los diferentes aspectos climáticos en nuestro patrimonio industrial, para su mejor conservación y así permitir nuevos usos en diferentes contextos futuros. El principio interdisciplinar de la cooperación multiherramienta no es sólo un esquema complejo de proceso de diseño, sino también una clave educativa en la protección del patrimonio industrial. Es necesario comprender a fondo cómo el sol afecta al clima, al microclima y al clima interior en los edificios. Este es un aspecto fundamental porque tiene influencia a largo y a corto plazo y necesitamos saber cómo interactúan con los efectos antropogénicos sobre las tres mencionadas escalas del clima. Nos estamos refiriendo a la particularización del cambio climático. También es importante comprender los factores de la variabilidad climática para manejar una base sobre la que predecir situaciones futuras.

Centrándonos en La Trinidad, con la realización de todos los gráficos y su posterior análisis, podemos verificar algunas deficiencias y cómo podrían resolverse. La simulación se basa en dos fechas críticas del calendario, el 21 de junio y el 21 de diciembre, los solsticios de verano e invierno, respectivamente. Como es sabido, estos son los días en que los períodos de radiación solar son más largos y más cortos, y, por lo tanto, los días de más y menos luz solar; así mismo, los ángulos de inclinación solar son los más extremos y producen los dos casos más dispares de incidencia posible (Figura 6). Este análisis, realizado a modo de testeo del caso, debe ser más profuso para un estudio completo de las implicaciones solares, abarcando un número mayor de fechas representativas y horas del día. Sin embargo, el proceso es el mismo y queda aquí suficientemente representado.

En primer lugar, hay que mencionar la escasez de luz natural en el espacio principal, con algunos picos cerca de las ventanas, dependiendo de la hora, dentro de un nivel general pobre. Esto supone el uso de iluminación artificial casi obligatoriamente para poder realizar cualquier tipo de actividad con requerimientos de visión suficiente en el futuro. También se ha observado que, debido al diseño

ACE, 15 (43) CC BY-ND 3.0 ES | UPC Barcelona, España | Fábricas de resiliencia. Una oportunidad para el patrimonio 10 industrial: el caso de La Trinidad. DOI: http://dx.doi.org/10.5821/ace.15.43.9192 
actual de las cubiertas, la luz apenas penetra en el edificio. Durante el periodo estival, la luz es casi cenital, por lo que las naves reciben muy poca luz natural. En invierno, sin embargo, la luz incide con un ángulo más horizontal y, por lo tanto, se alcanza una iluminación más distribuida, pero en cualquier caso insuficiente. Se alcanzan niveles de luminancia (lux) que sólo permiten actividades de precisión limitada, aunque pueden ser suficientes para cierto tipo de usos.

Figura 6. Simulación solar para iluminación en ambos solsticios. Situación actual
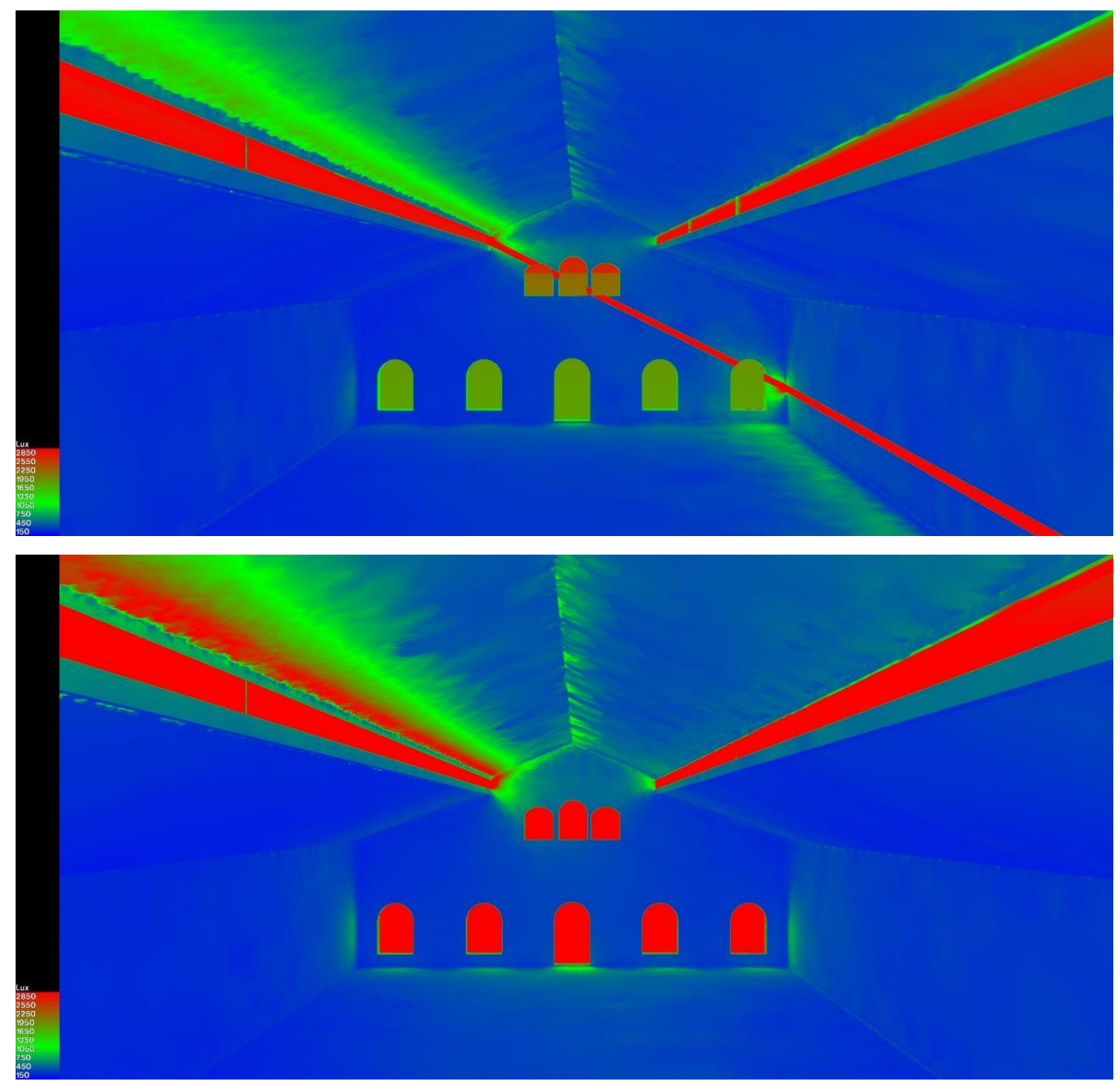

Unidad lux. Invierno-arriba/verano-abajo. Mediodía. Modelo 3D y simulación elaborada por los autores.

Sobre la base de estos resultados, hemos modelado una abertura en cubierta orientada al Sur (Figura 7) como una prueba de las posibilidades del volumen. Se puede observar cómo este caso ofrece resultados mucho mejores en términos de iluminación natural $y$, consecuentemente, puede suponerse que también de ganancia solar, con una iluminación significativamente más alta y una distribución más regular de los niveles de luz para cualquier situación durante el año. Una estrategia de diseño precisa debería simular la situación de iluminación adecuada para la actividad que se prevea y las obstrucciones de luz adecuadas para evitar el sobrecalentamiento en los meses de cálidos y el deslumbramiento no deseado según el uso proyectado. Dichos elementos de control no 


\section{ACE Architecture, City and Environment}

están incluidos en esta simulación, que se limita a un cambio genérico en el volumen y sólo explora las posibilidades del objeto arquitectónico como ejemplo de la operativa del método.

Figura 7. Simulación solar para iluminación en ambos solsticios. Situación modificada
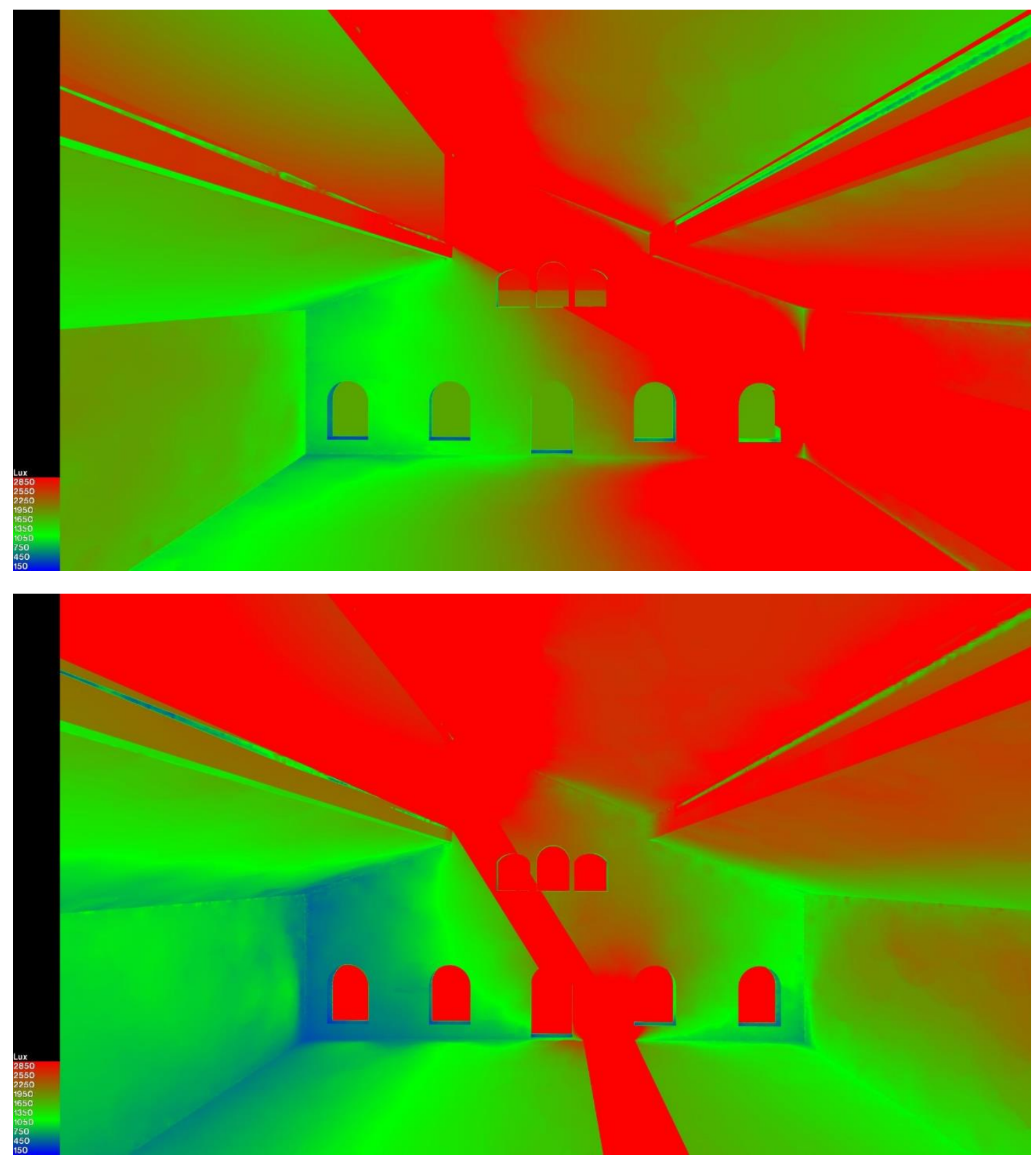

Unidad lux. Invierno-arriba/verano-abajo. Mediodía. Modelo 3D y simulación elaborada por los autores.

Este es sólo un ejemplo del tipo de procedimiento que debe verificarse en los procesos de control que proponemos durante la fase de simulación y no reproduce las posibilidades de la evaluación bioclimática completa. Muestra la forma en que una fase de simulación debe ayudar a intervenir de manera que se modele el edificio en una forma que produzca su integración en un sistema resiliente que garantice un largo período de uso para el propósito proyectado, con el objetivo de adecuación funcional, sostenibilidad ambiental (en términos de energía para este caso) y confort.

ACE, 15 (43) CC BY-ND 3.0 ES | UPC Barcelona, España | Fábricas de resiliencia. Una oportunidad para el patrimonio industrial: el caso de La Trinidad. DOI: $\underline{\text { http://dx.doi.org/10.5821/ace.15.43.9192 }}$ 


\subsection{Fase de materialización: Propuesta de resiliencia ambiental}

Después de la simulación, obtenemos los datos y el conocimiento para hacer frente a las decisiones que conducen a una solución arquitectónica adecuada. Pero esto es parte del objetivo del proceso. Como se mencionó anteriormente, esta metodología obtiene un equilibrio entre la realidad física (confort y resiliencia) por un lado y el factor social (funcionamiento adecuado de las intervenciones) por otro. No sólo es esencial que el edificio sea pasivo, un centro de producción y un modelo de rehabilitación integral, sino que tiene que representar un cambio fundamental de paradigma en la responsabilidad de los ciudadanos con respecto a su ciudad. La fábrica tiene que constituir un polo de educación y conciencia ambiental (Figura 8).

Figura 8. Ideograma de la propuesta de resiliencia ambiental mediante la integración de usos y estrategias
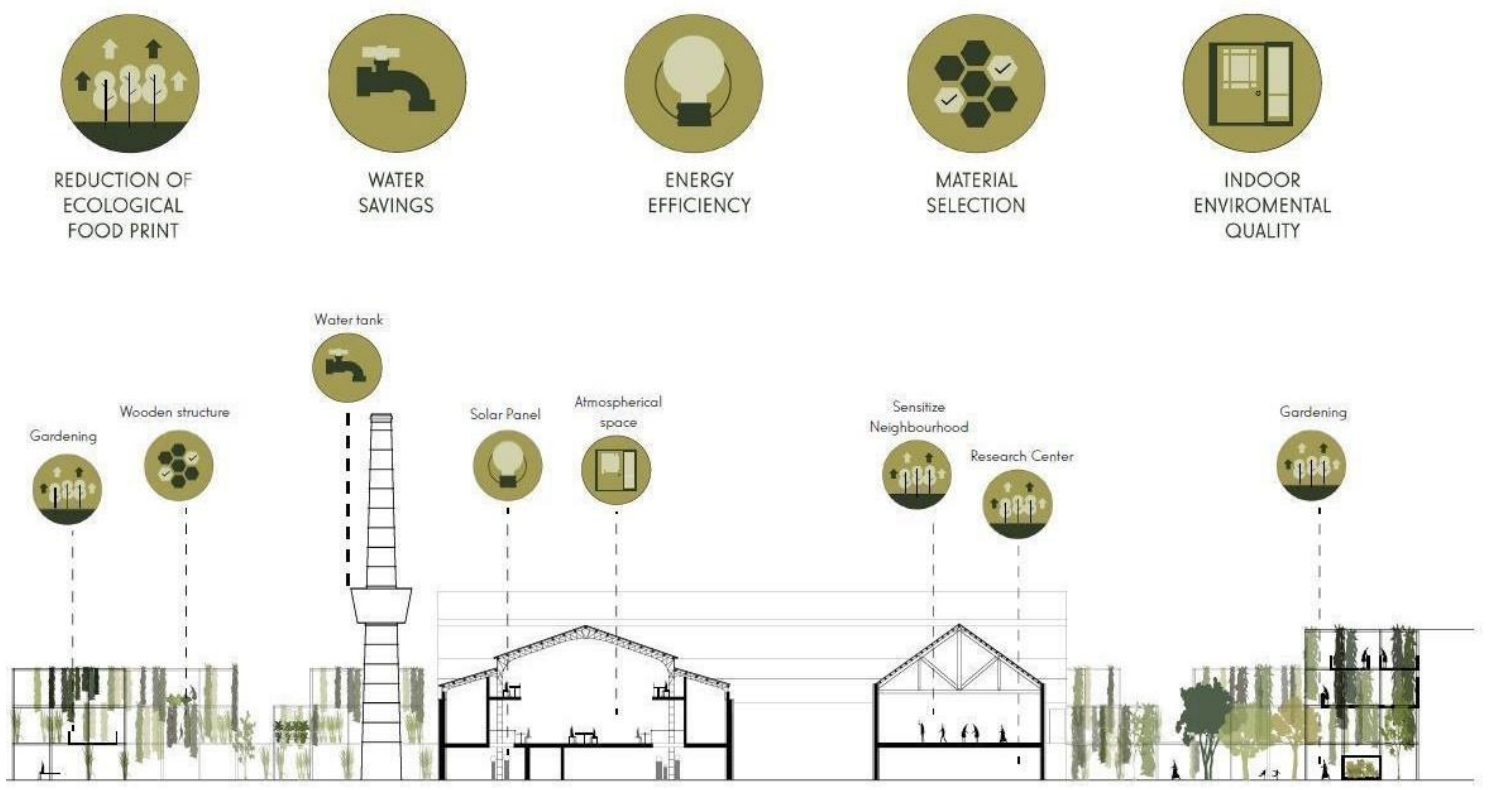

Fuente: Autores y equipo de investigación.

El cambio se explica a través de dos escalas, una global y otra específica. La global propone promover la producción local, el uso de materiales naturales, la aplicación de técnicas bioclimáticas, según los resultados de la simulación previa, y la búsqueda de un balance energético cero (o positivo), reduciendo drásticamente la demanda e integrando las energías renovables (producción de energía), y el principio de la cuna a la cuna, debido a Braungart \& McDonoug (2002). El paradigma en la escala específica se centra en reducir la huella ecológica de la intervención y el uso futuro del conjunto, ahorrar agua y energía a través de un proyecto pasivo y la selección de materiales que promueva un ambiente interior saludable. Cualquier propuesta de intervención tiene que pasar por conceptos básicos para la regeneración urbana y social del entorno de la Fábrica, así como el propio edificio, que sirven para sentar las bases de una metodología de intervención que pueda extrapolarse a otros casos, como se desarrolla en este texto.

El último pilar esencial es la conversión de la fábrica en un generador de resiliencia ambiental, aportando así valores que podrían servir de ejemplo para el proceso de transición del barrio y la ciudad. La mencionada integración de la vegetación como sumidero de CO2 y mitigador de la isla de calor urbana, por ejemplo, no sólo es esencial para que el edificio sea pasivo, un centro de producción y un modelo de rehabilitación integral, sino que tiene que representar un cambio fundamental de 
paradigma en la responsabilidad de los ciudadanos con respecto a su ciudad. Esta visión resiliente debe fomentar una nueva concepción del espacio público y del conjunto de elementos que constituyen su materialización: vegetación, agua, pavimentos, mobiliario urbano y elementos complementarios. Hay un nuevo objetivo primordial para la ciudad y es hacerla vivible en las nuevas condiciones climáticas que son previsibles, como se ejemplifica para nuestro caso en la Figura 9. Este argumento es desarrollado en detalle por Saeed et al. (2018).

Figura 9. La estrategia verde contra la burbuja de calor urbano
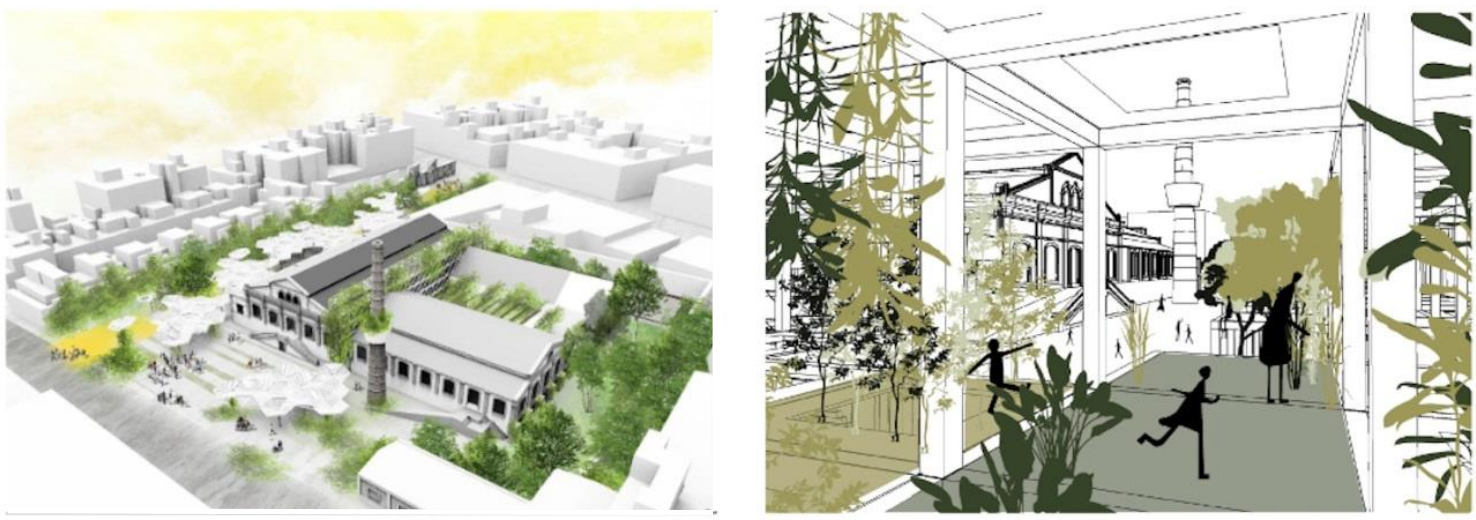

Fuente: Autores y equipo de investigación. Vista general (Izq.) y detalle de diseño de los espacios urbanos (der.).

Sólo un enfoque integrador como se muestra en este estudio, puede reinventar una ciudad resiliente, capaz de hacer frente con éxito a las incertidumbres económicas, sociales y climáticas de un futuro inmediato. Parafraseando al filósofo Watsuji (1961), la ciudad no existe sin el clima, así como el clima no existe sin la ciudad (donde nosotros decimos "ciudad" Watsuji decía "historia"). La arquitectura que configura esa ciudad y en este caso, el tejido de la arquitectura industrial y residencial, existe para ser un filtro entre el clima y los seres humanos que la habitan. Si fallamos en la recuperación y potenciación de este valor, nuestro esfuerzo en términos de resiliencia habrá sido en vano.

\section{Conclusiones}

Como hemos examinado y ejemplificado en esta investigación para el patrimonio industrial, podemos determinar que cada proceso de rehabilitación, en el sentido más amplio del término "rehabilitar", tiene un patrón de trabajo intrínseco que debe abordar las diferentes perspectivas que implica la intervención. Estas directrices pertenecen a un procedimiento genérico, sin entrar en las particularidades que presenta cada caso, cuya consideración será necesaria al abordar dichos casos concretos.

En primer lugar, tenemos el contexto urbano donde se inserta, que determinará su relación con el lugar, la parcela y la población local. No podemos olvidar que estos edificios son en su mayoría de propiedad privada, lo que suele implicar la negación de la realidad circundante a través del cierre de la parcela, por lo que habrá que reconectar el diálogo ciudad-edificio para lograr la relación adecuada.

En segundo lugar, es necesaria la previsión de un programa que permita la reactivación social del conjunto, de tal manera que vuelva a ser un foco de actividad, así como integrar la memoria del lugar en varios planos con estos usos, desde la estructuración social hasta la memoria de la producción como patrimonio intangible. 
En tercer lugar, operamos con el propio edificio, recordando que el potencial del patrimonio industrial va mucho más allá de su mera consideración como contenedores, pero haciendo de su fuerte huella una línea base del proyecto, para elaborar propuestas más complejas y completas que de lo contrario no serían posibles.

Por último, aunque no menos importante, está la componente ambiental, a la que presta una atención especial esta investigación. Nos encontramos con que estos edificios no sólo se han pensado sin tener en cuenta la lógica climática, sino que han sido un foco de contaminación dentro de la ciudad y sus alrededores.

La reutilización del patrimonio industrial debe potenciar sus valores medioambientales como una de las razones de ser de los edificios en los que intervenimos. Gracias a las herramientas científicas y la simulación, el potencial ambiental de estas arquitecturas, que a menudo se intuye, pero casi nunca se cuantifica, se puede conocer en detalle. Dar valor al vacío, el espacio verdaderamente sentido y habitado por los seres humanos, significa revalorizar el patrimonio industrial, ahora constituido por estructuras disfuncionales en gran medida, para darles una nueva vida. Para la arquitectura y el urbanismo este factor es de gran importancia, ya que se puede concluir que la forma arquitectónica y urbana es un factor primordial para los valores psicrométricos y ambientales.

El objetivo es ir más allá de la sostenibilidad, a una re-conceptualización donde la antigua industria abandonada se convierta en fábricas de resiliencia para la sociedad que anteriormente las construyó y ahora decide intervenir y recuperarlas. Del mismo modo que una vez trajo riqueza a una red social, proponemos repensarlas como foco de los nuevos recursos que ahora necesitamos. Estas rehabilitaciones representan la oportunidad de revertir la lógica de la operación original, consiguiendo una gestión positiva de los recursos, compensando los años de contaminación con intervenciones del menor impacto posible en múltiples campos y, en última instancia, generando focos de resiliencia para la ciudad y la sociedad donde renacen.

\section{Agradecimientos}

Deseamos agradecer su colaboración en alguna parte del estudio a: Luis González de Boado, Belén Rey, Alejandro Zújar y los alumnos participantes en el Taller Re-Fact 2017 sobre Patrimonio Industrial.

\section{Autoría}

Ambos autores suscriben la totalidad del trabajo y su conceptualización desde la ecología. SánchezMontañés ha desarrollado los aspectos relacionados con el estudio patrimonial y la visión integral de resiliencia y Castilla es el autor de la metodología aplicada y el desarrollo de la fase de simulación.

Conflicto de intereses: Los autores declaran que no hay conflicto de intereses.

\section{Bibliografía}

Andrade, J.B. y Brangança, L. (2011). Analysis of the impacts of economic and social indicators to sustainability assessment. En Proceedings of the International Conference Sustainability of Constructions - Towards a better built environment (p. 163-168). Innsbruck, Austria. Recuperado de https://pdfs.semanticscholar.org/1a21/e708ff38f745a502fc8b57762408193a1cc2.pdf? ga=2.16325580 4.1735408487.1590070403-258945694.1590070403 
Attia S.; Hensen J.; Beltran I. y De Herde A. (2012). Selection criteria for building performance simulation tools: contrasting architects' and engineers' needs. Journal of Building Performance Simulation 5(3), 155-159. DOI: https://doi.org/10.1080/19401493.2010.549573

Ayuntamiento de Madrid. (2010). Metodología de Evaluación para el Programa de Ayudas a las Actuaciones de Rehabilitación para la Mejora de la Sostenibilidad y Eficiencia Energética de las Edificaciones.

Bender, B. (2001). Landscapes on-the-move. Journal of Social Archaeology, 1(1), 75-89. DOI: https://doi.org/10.1177/146960530100100106

Braungart, M. y McDonough, W. (2002). Cradle to Cradle: Remaking the Way We Make Things. Nueva York, EEUU: North Point Press.

Campos-Sánchez F.S.; Abarca-Álvarez F.J. y Domingues A. (2018). Sostenibilidad, planificación y desarrollo urbano: en busca de una integración crítica mediante el estudio de casos recientes. ACE: Architecture, City and Environment, 12(36), 39-72. DOI: http://dx.doi.org/10.5821/ace.12.36.5145

Castilla, M. V. (2016). Integrated Multi-Tool System in Creative Design for Solar Radiation. En Z. Car y J. Kudlacek (Ed.), IN-TECH 2016 International Conference on Innovative Technologies. Praga, República Checa: Faculty of Engineering University of Rijeka.

Castilla, Manuel V. (2018). Climate Responsive Design Simulation and Modelling for Industrial Heritage. Modelling and Simulation in Engineering. Vol. 2018. DOI: https://doi.org/10.1155/2018/5253100

Fiocchi, C.; Hoque, S. y Shahadat, M. (2011). Climate Responsive Design and the Milam Residence. Sustainability, 3(11), 2289-2306. DOI: https://doi.org/10.3390/su3112289

Fiksel, J. (2006). Sustainability and resilience: toward a systems approach. Sustainability: Science, Practice \& Policy 2(2), 14-21. DOI: https://doi.org/10.1080/15487733.2006.11907980

Givoni, B. (1969). Man, climate and architecture. Londres, Reino Unido: Elsevier Publishiing.

Hernández Martínez, A. (2007). El reciclaje de la arquitectura industrial. En Patrimonio Industrial y la Obra Pública. Actas. Jornadas. (pp. 29-52). Zaragoza, España: Servicio de Publicaciones del Gobierno de Aragón.

Higón Calvet, J.L. (2003). Contribuciones al estudio del asoleo geométrico. Procedimientos para el cálculo del factor de obstrucción solar. Aplicaciones. (Tesis Doctoral). Universidad Politécnica de valencia, valencia.

Hopkins, R. (2008). The transition handbook. Totnes, Reino Unido: Transition Network.

Iwaro, J. y Mwasha, A. (2014). The impact of sustainable building envelope design on building sustainability using Integrated Performance Model. International Journal of Sustainable Built Environment, 2(2), 153-171. DOI: https://doi.org/10.1016/j.ijsbe.2014.03.002

Kevseroğlu, D. (2014). The evaluation of the integration of industrial heritage areas to urban landscape: The case study of Sumerbank Kayseri cotton factory. A/Z ITU, 12(1), 31-36. Recuperado de https://www.az.itu.edu.tr/azvol12no1web/6-\%20kevseroglu-kubat-1201.pdf

ACE, 15 (43) CC BY-ND 3.0 ES | UPC Barcelona, España | Fábricas de resiliencia. Una oportunidad para el patrimonic 16 industrial: el caso de La Trinidad. DOI: http://dx.doi.org/10.5821/ace.15.43.9192 
Landorf, C. (2009). A framework for sustainable heritage management: a study of UK industrial heritage sites. International Journal of Heritage Studies, 15(6), 494-510. DOI: https://doi.org/10.1080/13527250903210795

Lombera J.T. y Rojo J.C. (2010). Industrial building design stage based on a system approach to their environmental sustainability. Construct. Build. Mater, 24(4), 438-447. DOI: https://doi.org/10.1016/j.conbuildmat.2009.10.019

Manley, G. (1958). On the frequency of snowfall in metropolitan England. Quarterly Journal of the Royal Meteorological Society, 84 (359), 70-72. DOI: https://doi.org/10.1002/qj.49708435910

Minslow J.C., Hunt, E., y Piper, S. (2001). A global applicable model of daylight solar irradiance estimated for air temperature and precipitation data. Ecological Modelling, 143, 227-243. Recuperado de https://hrsl.ba.ars.usda.gov/ERHunt/winslow ecomod2001.pdf

Oglethorpe, M. (2013). Industrial heritage: Realising the value of our historic assets. En P. Hájek et al. (Eds.), Central Europe Towards Sustainable Building: Sustainable Building and Refurbishment for Next Generations, (pp. 249-252). Praga, República Checa: CESB. Recuperado de http://cesb.cz/cesb13/index.html

Olmos, D. (2013). Programas de Simulación: Análisis comparativo de 3 Software de Simulación Energética Aplicados a un Proyecto construido y monitorizado. (Proyecto Fin de Máster Oficial) Universidad Politécnica de Cataluña, Barcelona.

Rosen, M.A. (2012). Engineering Sustainability: A Technical Approach to Sustainability, Sustainability, 4(9), 2270-2292. DOI: https://doi.org/10.3390/su4092270

Rubio, C. Pulido, J. Sánchez-Montañés, B. (2015). A simplified simulation model for predicting radiative transfer in long street canyons under high solar radiation conditions. Energies, 8(12), 13540-13558. DOI: https://doi.org/10.3390/en81212383

Saeed, E.; Azmy, N. y Omar, N. (2018). The role of the landscape elements to improve the urban spaces environmental performance. ACE: Architecture, City and Environment, 12(36), 91-110. DOI: http://dx.doi.org/10.5821/ace.12.36.4851

Sánchez-Montañés, B. (2011). The skin of the memory. En E. Farini (Ed.) Time and Cities (pp. 133-141). Madrid, España: Universidad Francisco de Vitoria

Sobrino, J. (2018). La Trinidad glass factory: a transparent heritage. In B. Sánchez-Montañés, L. González y M. Castilla, (Eds.), Obsolescence and opportunity in the forgotten industry: The glass factory La Trinidad (pp. 23-70). Sevilla, España: TICCIH.

Sobrino, J. (2015). Fábricas que cierran: la paradoja de lo productivo desocupado. En Pelegrín, M. y Pérez, F. (Eds.), Arquitectura dispuesta: preposiciones cotidianas = Architecture set: everyday life prepositions (pp. 182-193). Sevilla, España: Universidad de Sevilla.

Sobrino, J. (2010). Los paisajes históricos de la producción en Sevilla (Proyecto Guía del Paisaje Histórico Urbano). Sevilla, España: Universidad de Sevilla.

Trachana, A. (2008). Arqueología Industrial y restauración ambiental. Buenos Aires, Argentina: Nobuko.

ACE, 15 (43) CC BY-ND 3.0 ES | UPC Barcelona, España | Fábricas de resiliencia. Una oportunidad para el patrimonio 
Vilches, A.; García-Martínez, A. y Sánchez-Montañés, B. (2016). Life cycle assessment (LCA) of building refurbishment: A literature review. Energy and Buildings, 135, 286-301. DOI: https://doi.org/10.1016/j.enbuild.2016.11.042

Watson, M. (2012). Adaptive re-use and Embodied Energy. En Douet, J (ed), Industrial Heritage Retooled: The TICCIH Guide to Industrial Heritage Conservation, (pp. 136-141). Lancaster, Reino unido: Carnegie Publishing.

Watsuji, T. (1961). A climate: A philosophical study. Tokio, Japón: Japanese National Commission for UNESCO.

Wood, T. (2010). Climate Analysis Using Autodesk Ecotect Weather Tool and Solar Tool. Trabajo presentado en AIA Montana Annual Fall Conference. Helena, USA. Recuperado de https://docplayer.net/41239349-Part-3-climate-analysis-using-autodesk-ecotect-weather-tool-andsolar-tool.html 